\title{
APPLICATIONS OF NEUTRON POWDER DIFFRACTION
}

\author{
Erich H. Kisi \\ School of Engineering, The University of Newcastle, \\ Australia \\ Christopher J. Howard \\ School of Engineering, The University of Newcastle, \\ Australia
}




\section{Contents}

Preface ix

Acknowledgements $\quad$ xi

Image Acknowledgements $\quad$ xii

Glossary of symbols $\quad$ xiii

1 Introduction to neutron powder diffraction 1

$\begin{array}{ll}\text { 1.1 What is neutron powder diffraction? } & 1\end{array}$

1.2 The role of neutron powder diffraction 2

1.3 Milestones in the development of neutron powder diffraction 3

2 Theory - the bare essentials $\quad 18$

$\begin{array}{ll}2.1 & \text { Neutrons for diffraction } \\ 2.2 & 18\end{array}$

2.2 Samples for diffraction - the structure of condensed matter 20

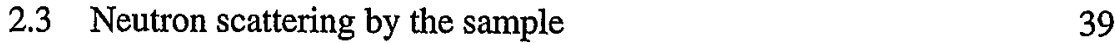

$2.4^{*}$ The powder diffraction pattern 49

3 Basic instrumentation and experimental techniques 65

3.1 Where to find neutron powder diffraction facilities 65

$\begin{array}{ll}3.2 \text { Constant wavelength neutron diffractometers } & 70\end{array}$

$\begin{array}{lll}3.3 & \text { TOF neutron diffractometers } & 77\end{array}$

3.4 Comparison of CW and TOF diffractometers 80

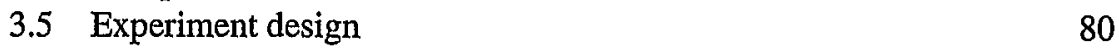

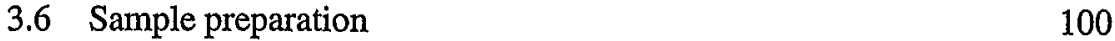

4 Elements of data analysis $\quad \mathbf{1 0 6}$

$\begin{array}{ll}4.1 \text { Preliminaries } & 106\end{array}$

$\begin{array}{ll}4.2 \text { Visual inspection } & 109\end{array}$

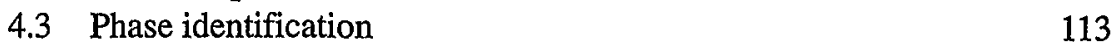

$\begin{array}{ll}4.4 & \text { Unit cell parameters } \\ \end{array}$ 
$\begin{array}{lll}4.5 & \text { Peak shapes and widths } & 124\end{array}$

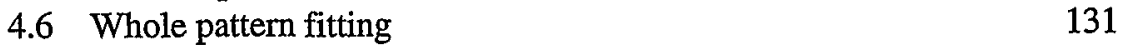

5 Crystal structures 134

5.1 Neutron powder diffraction and crystal structures 134

5.2 More crystallography - description of crystal structures 135

5.3 Reflection conditions and space group determination 146

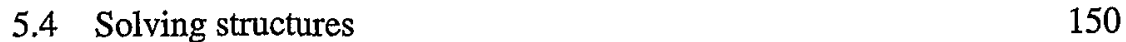

5.5 Structure refinement - the Rietveld method 155

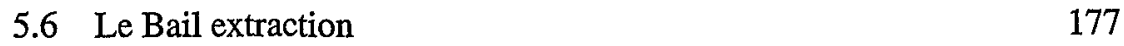

5.7 Practical considerations in structure refinement 178

$\begin{array}{lll}5.8 & \text { Structure solution and refinement - examples } & 182\end{array}$

6 Ab initio structure solution $\quad 192$

$\begin{array}{ll}6.1 \text { Introduction } & 192\end{array}$

6.2 Unit cell determination (powder pattern indexing) 193

$\begin{array}{ll}6.3 \text { Intensity extraction } & 205\end{array}$

6.4 Structure solution $\quad 212$

6.5 Advanced refinement techniques 232

$\begin{array}{ll}6.6 \text { Looking ahead } & 249\end{array}$

7 Magnetic structures $\quad 251$

$\begin{array}{ll}7.1 \text { Introduction } & 251\end{array}$

7.2 Crystallography and symmetry of magnetic structures 252

$\begin{array}{ll}7.3 \text { Magnetic scattering and diffraction } & 260\end{array}$

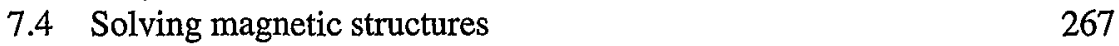

$\begin{array}{lll}7.5 & \text { Recent examples } & 273\end{array}$

8 Quantitative phase analysis $\quad 284$

$\begin{array}{ll}8.1 \text { Introduction } & 284\end{array}$

$\begin{array}{ll}8.2 \text { Theory } & 285\end{array}$

8.3 Individual peak methods 287

8.4 Whole pattern analysis $\quad 292$

8.5 Evaluation of the techniques 293

8.6 Practical examples 294

9 Microstructural data from powder patterns 308

$\begin{array}{lll}9.1 \text { Introduction } & 308\end{array}$

$\begin{array}{lll}9.2 & \text { Particle size } & 309\end{array}$

$\begin{array}{ll}9.3 \text { Microstrains } & 330\end{array}$

9.4 Combined size and strain broadening $\quad 340$ 
9.5 Chemical and physical gradients 346

9.6 Line defects - dislocation broadening 358

9.7 Plane defects and stacking disorder 368

$\begin{array}{lll}9.8 \text { Texture } & 374\end{array}$

10 Diffuse scattering - thermal, short-range order, gaseous, liquid, and amorphous scattering

10.2 Thermal diffuse scattering $\quad 382$

10.3 Short-range order scattering 385

10.4 Scattering from gases, liquids, and amorphous solids 395

11 Stress and elastic constants 403

11.1 Stresses, strains, and elastic constants in nature and industry 403

11.2 Influence of elastic strains on the powder diffraction pattern 414

11.3 Neutron diffraction residual stress analysis 420

11.4 Determination of single crystal elastic constants from polycrystalline samples

12 New directions $\quad \mathbf{4 4 3}$

12.1 Introduction 443

12.2 Neutron sources 443

12.3 Components 444

12.4 Diffractometers 446

12.5 Data analysis 449

12.6 New problems for study by neutron powder diffraction 453

12.7 Closing remarks $\quad 458$

$\begin{array}{ll}\text { Appendix } 1 & 459\end{array}$

Appendix 2 462

$\begin{array}{ll}\text { References } & 463\end{array}$

$\begin{array}{ll}\text { Index } & 481\end{array}$ 